\title{
Corticosteroid Treatment Guided by Peripheral Blood Mononuclear Cell Counts in Cerebral Amyloid Angiopathy-related Inflammation: A Case Report
}

Ya Li

Affiliated Hospital of Guizhou Medical University

ChunTing Liu

Guizhou Center for Disease Control and Prevention

ShiPeng Guo

Affiliated Hospital of Guizhou Medical University

AnNi Zhang

Affiliated Hospital of Guizhou Medical University

XuLing Wu

Affiliated Hospital of Guizhou Medical University

Dian He ( $\nabla$ hedian@gmc.edu.cn )

Affiliated Hospital of Guizhou Medical University https://orcid.org/0000-0003-0550-6249

\section{Case report}

Keywords: cerebral amyloid angiopathy-related inflammation, rapidly progressive dementia, lobar microbleeds, apolipoprotein E, Aß42, corticosteroid treatment, $B$ lymphocytes, case report

Posted Date: March 5th, 2020

DOI: https://doi.org/10.21203/rs.3.rs-16072/v1

License: (c) (1) This work is licensed under a Creative Commons Attribution 4.0 International License. Read Full License 


\section{Abstract}

Background: Cerebral amyloid angiopathy-related inflammation (CAA-I), a rare variant of cerebral amyloid angiopathy, is one of treatable causes of rapidly progressive dementia. CAA-I usually occurs in the elderly and corticosteroids are most often used for treatment. Thus it is more necessary to develop an individualized dosing regimen to maximize efficacy yet minimize adverse effects of corticosteroids. We report a CAA-I patient successfully treated by corticosteroid therapy guided by dynamic monitoring of peripheral blood mononuclear cell (PBMC) count.

Case presentation: A 68-year-old female presented with subacute step-wise cognitive decline. Medical history and current examination revealed multiple risk factors for cerebral amyloid angiopathy, including apolipoprotein E $\varepsilon 4 / \varepsilon 4$ genotype, hyperhomocysteinemia, hypertension, and chronic renal failure, while brain susceptibility-weighted magnetic resonance imaging revealed diffuse lobar microbleeds associated with extensive lesions disturbed throughout cerebral grey and white matter on T1- and T2-weighted MRI. The CD19+ B-cell fraction of PBMCs was markedly elevated, as were inflammatory markers such as erythrocyte sedimentation rate and C-reactive protein. Cerebrospinal fluid total protein and T-tau levels were also elevated, while $A \beta 42$ was low and P-tau levels were normal. Corticosteroid treatment guided by dynamic CD19+ B-cell number monitoring reversed these inflammatory signs, imaging manifestations, and acute cognitive decline.

Conclusions: Corticosteroid treatment individualized by dynamic monitoring of PBMC CD19+ B-cell fraction may provide for maximum therapeutic efficacy with minimal adverse effects in patients with CAA-I.

\section{Background}

Cerebral amyloid angiopathy (CAA)-related inflammation (CAA-I) is a rare CAA subtype [1] characterized by reversible encephalopathy with acute or subacute cognitive decline, seizures, headache, and focal neurologic signs [2]. CAA-I can be divided into two subtypes, inflammatory CAA and amyloid- $\beta$ (AB)related angiitis, according to histopathology [2]. The majority of patients with CAA-I ( 80\%) respond to immunotherapy, but roughly $30 \%$ have symptomatic relapse [1]. Among the immunotherapies, corticosteroids are most often used for CAA-I treatment. Considering CAA-I usually occurs in the elderly, it is more necessary to develop an individualized dosing regimen to maximize efficacy yet minimize adverse effects of corticosteroid. Herein, we report a case of CAA-I successfully treated by corticosteroid therapy guided by peripheral blood mononuclear cell (PBMC) counts.

\section{Case Presentation}

A 68-year-old female presented with subacute step-wise cognitive decline and behavioral abnormalities for the previous eight months. She was diagnosed with chronic nephritis 30 years ago and with hypertension and chronic renal failure 1 year prior to the current presentation. The family history was 
unremarkable. Head computed tomography (CT) plain scan conducted at another hospital after current symptom onset revealed multiple punctate high-density lesions and extensive low-density lesions in both cerebral hemispheres. Accompanying magnetic resonance imaging (MRI) showed extensive hyperintense lesions on both T2-weighted images (T2WI) and fluid-attenuated inversion recovery (FLAIR) images without gadolinium enhancement on T1-weighted images (T1WI). Brain susceptibility weighted imaging (SWI) was not conducted at that time. The patient is illiterate and scored 14/30 on the Mini-Mental State Examination (MMSE). The diagnosis was undetermined at that time and no specific therapies were given except for antihypertensive agents.

On admission at our institution, blood pressure was $143 / 102 \mathrm{mmHg}$. The patient was fully alert but disoriented and cognitive function tests revealed subacute cognitive decline, with MMSE score of 4/30, Montreal Cognitive Assessment (MoCA) score of 0/30, and Activities of Daily Living (ADL) scale score of 44/64. Results of cranial nerve and sensory examinations were negative, and motor examination revealed positive palmomental reflex bilaterally without weakness or dystonia of limbs.

Routine blood tests revealed low hemoglobin $(97 \mathrm{~g} / \mathrm{L})$ and free triiodothyronine $(2.97 \mathrm{pmol} / \mathrm{L})$, and elevated urea nitrogen $(10.52 \mathrm{mmol} / \mathrm{L})$, creatinine $(221.83 \mu \mathrm{mol} / \mathrm{L})$, uric acid $(371.12 \mu \mathrm{mol} / \mathrm{L})$, brain natriuretic peptide $(2131.00 \mathrm{pg} / \mathrm{mL})$, homocysteine $(26.46 \mu \mathrm{mol} / \mathrm{L})$, erythrocyte sedimentation rate (ESR) $(120 \mathrm{~mm} / \mathrm{h})$, and C-reactive protein (CRP) $(28.35 \mathrm{mg} / \mathrm{L})$. The titers of thyroglobulin antibody (TGAb) (> $500.00 \mathrm{U} / \mathrm{ml}$ ) and thyroid peroxidase antibody (TPOAb) $(197.90 \mathrm{U} / \mathrm{ml})$ were elevated, as was CD19+ Bcell fraction among PBMCs (16.26\%). Serum anti-Ro-52 was positive, while all other serum autoantibody tests were negative (including anti-nuclear, anti-Sjogren syndrome antigen $A$ and antigen B, cytoplasmic antineutrophil, and anticardiolipin antibodies). Serum anti-human immunodeficiency virus antibodies and anti-Treponema pallidum antibodies were also negative. Serum cholesterol, triglyceride, coagulation function, tumor markers, folic acid, and vitamin B12 were within normal limits. Apolipoprotein E (APOE) genotype was $\varepsilon 4 / \varepsilon 4$, indicating elevated CAA risk. Cerebrospinal fluid (CSF) analysis revealed elevated levels of total protein $(80.00 \mathrm{mg} / \mathrm{dL})$ and T-tau $(583 \mathrm{pg} / \mathrm{ml})$, low Aß42 $(486 \mathrm{pg} / \mathrm{ml})$, normal nucleated cell count, and normal P-Tau (181p) $(60.42 \mathrm{pg} / \mathrm{ml})$. All TORCH panel assays were negative.

In accord with prior neuroimaging results, brain CT plain scans showed multiple punctiform hyperdensities in the bilateral lobes (Figure 1-A), and brain MRI scans showed extensive hypointense lesions on T1WI (Figure 1-B) and hyperintense lesions on T2WI (Figure 1-C) and FLAIR (Figure 1-D) images in bilateral cerebral hemispheres involving both grey and white matter. Diffusion-weighted imaging (DWI) results were unremarkable (Figure 1-E) but SWI scans revealed diffuse microbleeds mainly distributed in bilateral lobes and cerebellum (Figure 1-F). Head magnetic resonance angiography (MRA) revealed mild arteriosclerosis in bilateral internal carotid arteries and middle cerebral arteries. Carotid duplex ultrasound examination also revealed moderate stenoses $(50 \%-70 \%)$ of the left carotid bulb and right subclavian artery.

After informed consent was obtained from the patient's legal guardian, minimally invasive biopsy of affected white matter in the left occipitotemporal lobe was performed under stereotactic orientation. 
Hematoxylin and eosin staining of biopsy tissue revealed edema and glial cell proliferation, but no inflammatory infiltrations surrounding the blood vessels. No amyloid deposition in the vessel walls was found by Congo Red staining, under a polarizing microscope.

Based on these findings, the patient was diagnosed with probable CAA-related inflammation [3] and received intravenous methylprednisolone (IVMP) starting at $500 \mathrm{mg} / \mathrm{d}$, followed by oral prednisone with slow tapering guided by monitoring of PBMC counts (Figure 2). Felodipine (10 mg/d), irbesartan (150 $\mathrm{mg} / \mathrm{d})$, and metoprolol $(25 \mathrm{mg} / \mathrm{d}$ ) were also administered for hypertension and blood pressure was controlled at about $140 / 90 \mathrm{mmHg}$. Folic acid was orally supplemented to lower serum homocysteine and atorvastatin $(20 \mathrm{mg} / \mathrm{d})$ to prevent arteriosclerosis. Cognitive symptoms started to improve on the third day of IVMP treatment at $500 \mathrm{mg} / \mathrm{d}$ as indicated by increased MMSE (7/30, compared to 4/30 at presentation), while MoCA remained 0/30 and ADL was lower than on presentation (34/64). Blood CRP level returned to normal and erythrocyte sedimentation rate dropped but was still elevated $(80 \mathrm{~mm} / \mathrm{h})$ after the 5-day course of IVMP $500 \mathrm{mg} / \mathrm{d}$ (Figure 2). The patient was then treated with a 3-day course of IVMP $250 \mathrm{mg} / \mathrm{d}$ and a 3-day course of IVMP $120 \mathrm{mg} / \mathrm{d}$, after which cognitive symptoms and daily living function improved, with MMSE increasing to 10/30, MoCA increasing to 6/30, and ADL decreasing to 26 . Repeated MRI showed remarkable shrinkage of hyperintense lesions on FLAIR images (Figure 2). However, the CD19+ B-cell fraction among PBMCs was still markedly elevated at $45.33 \%$. Oral prednisone $60 \mathrm{mg} / \mathrm{d}$ was then administered with tapering of $5 \mathrm{mg}$ every three days.

When the dose of prednisone reached $30 \mathrm{mg}$ daily, retests revealed normal ESR, while hemoglobin was slightly lower $(95 \mathrm{~g} / \mathrm{L})$ and urea nitrogen $(16.64 \mathrm{mmol} / \mathrm{L})$, creatinine $(279.31 \mu \mathrm{mol} / \mathrm{L})$, uric acid $(694.76$ $\mu \mathrm{mol} / \mathrm{L})$, and homocysteine $(38.87 \mu \mathrm{mol} / \mathrm{L})$ slightly increased compared to measurements on admission. In addition, the MMSE and MoCA score continued to increase, reaching 14/30 and 9/30 respectively, and the ADL score further decreased to 20/64. Erythropoietin was then started at $4000 \mathrm{IU}$ three times per week, and oral vitamins B12 and B6 were added to lower homocysteine level. The prednisone tapering interval was changed to $5 \mathrm{mg}$ per week. When the dose reached $20 \mathrm{mg}$ daily, CD19+ B-cell fraction among PBMCs was reduced to $30.75 \%$, hemoglobin increased to $105 \mathrm{~g} / \mathrm{L}$, and levels of creatinine $(247.07$ $\mu \mathrm{mol} / \mathrm{L})$ and uric acid $(681.55 \mu \mathrm{mol} / \mathrm{L})$ slightly reduced. Repeated MRI scans showed further shrinkage of hyperintense lesions on T2WI images (Figure 2). Subsequently, the dose of prednisone was maintained at 20 mg daily.

After one month of $20 \mathrm{mg}$ daily prednisone, the CD19+ B-cell fraction was normal (9.18\%), hemoglobin was higher but still below normal $(107 \mathrm{~g} / \mathrm{L})$, homocysteine $(16.62 \mu \mathrm{mol} / \mathrm{L})$ was normal, and both creatinine $(294.85 \mu \mathrm{mol} / \mathrm{L})$ and uric acid $(709.46 \mu \mathrm{mol} / \mathrm{L})$ were slightly higher compared to the previous measurement. There was further improvement in cognition as indicated by a MMSE of 20/30 and a MoCA of 13/30. Daily living function returned to normal, with the lowest score of 14/64 on ADL. Tapering of prednisone was then restarted at $5 \mathrm{mg}$ every three days until reaching a maintenance does of $5 \mathrm{mg}$ daily. Oral sodium bicarbonate was also administered to reduce uric acid. One month later, cognitive function was still stable, with a score of 22/30 on the MMSE and a score of MoCA of 13/30. Repeated MRI scans showed some residual hyperintensities in the deep white matter on FLAIR images (Figure 2). 
The CD19+ B-cell fraction among PBMCs was still in the normal range (10.53\%). The dose of prednisone was then changed to $5 \mathrm{mg}$ every other day for prevention of inflammation relapse. At one-year follow-up, the patient showed no signs of inflammation relapse or symptomatic intracerebral hemorrhage (ICH). The detailed clinical course as well as longitudinal changes in CD19+ B-cell and CD4+Th-cell fractions, cognition scale scores, and glucocorticoid therapy regimen are shown in Figure 2.

\section{Discussion And Conclusions}

This case exhibited the typical clinical and neuroimaging manifestations of CAA-I, but presented with only one of the major symptoms (rapidly progressive dementia) without seizures, headaches, or focal neurologic deficits. However, brain CT scans at the time of dementia onset showed multiple lobar hemorrhages, a frequent manifestation of CAA in patients over the age of 55. Neuroinflammation due to CAA should be highly suspected in rapid onset dementia cases with patchy or confluent white matter hyperintensities on T2WI. Extensive lobar microbleeds on SWI acquired at our institution further supported probable CAA-I according to clinicoradiological criteria [3]. It is well known that both SWI and gradient-echo MRI are sensitive to hemorrhage, especially microhemorrhage and chronic-phase hemorrhage. Indeed, hypointensities on SWI are widely considered a biomarker for CAA reflecting underlying brain lesions [4].

We also performed a brain biopsy to determine the exact pathological subtype of CAA-I but this yielded no definitive results. This false-negative is not unusual, however, as amyloid-laden vessels and other signs may be sparse in certain regions depending on disease stage and so are easily missed. Indeed, only $57 \%$ of brain biopsies in patients with dementia are diagnostic [5]. There is a general region-specific temporal pattern to vessels affected by $A \beta$ accumulation in CAA. Initially, arteries of the leptomeninges show signs of pathology, followed shortly by penetrating arterioles in the neocortical grey matter. Vessels to the posterior aspects of the brain are particularly impacted, most severely in the occipital lobe and parietal region, although in some cases the frontal cortex is the primary site of CAA pathology. In the next stage, vessels in the olfactory cortex, hippocampus, and cerebellum may be affected. Finally, deep grey and white matter are involved [6]. The biopsy site in this case was the deep white matter of the left occipitotemporal lobe, a region likely affected by inflammation rather than amyloid-laden vessels. Despite failing to obtain direct evidence for amyloid deposits, histopathology and the absence of gadolinium enhancement on T1WI ruled out brain tumor, viral and autoimmune encephalitis, and primary angiitis of the central nervous system.

The case patient demonstrated elevated ESR, CRP, and CSF protein with no signs of infection. It has been reported that about $33 \%$ of CAA-I cases exhibit elevated levels of such inflammatory markers in the peripheral blood, while $45 \%$ show pleocytosis in the CSF, and $71 \%$ show mildly elevated protein in the CSF [2]. It has also been reported that half of CAA patients meet the pathological diagnostic criteria for Alzheimer's disease (AD) [7]. However, this case demonstrated low CSF Aß42, high T-tau, and normal Ptau (181p), further supporting CAA and excluding the possibility of AD, which is associated with high CSF P-tau. A previous study also documented lower CSF A 340 and AB42 concentrations in CAA compared to 
both controls and patients with $A D$ [8]. Furthermore, the presence of microbleeds was reported to be associated with lower CSF AB42 in AD and vascular dementia, especially in apolipoprotein $E \& 4$ carriers [9].

This case carried an APOE epsilon $4(\varepsilon 4)$ /epsilon $4(\varepsilon 4)$ genotype, which further supported a diagnosis of CAA as there is a dose-dependent association between APOE $\varepsilon 4$ and sporadic CAA [10]. Patients carrying the APOE epsilon $4(\varepsilon 4)$ or epsilon $2(\varepsilon 2)$ alleles are at greater risk for CAA-related hemorrhage than those with the common APOE epsilon $3(\varepsilon 3)$ allele [11]. Among the vessels affected by A $\beta$ deposition, capillary involvement is strongly associated with the APOE $\varepsilon 4$ allele [12]. Moreover, the APOE $\varepsilon 4 / \varepsilon 4$ genotype is strongly associated with the occurrence of inflammation induced by CAA [1].

This case also exhibited hyperhomocysteinemia, which may have increased the disease load. Animal studies have demonstrated that high levels of homocysteine can lead to more severe CAA and parenchymal $A \beta$ deposition [13]. Of note, this case also showed markedly elevated levels of TGAb and TPOAb, so Hashimoto encephalopathy (HE) should be considered in the differential diagnosis because this disease also causes subacute onset cognitive dysfunction and confusion with altered consciousness. However, MRI findings were inconsistent with the neuroimaging manifestations of $\mathrm{HE}$. In $\mathrm{HE}$, most MRI sequences are normal, but patients may demonstrate cerebral atrophy or nonspecific signal abnormalities in the subcortical white matter on T2WI [14].

This case also had long histories of hypertension and chronic renal failure, both of which can aggravate microbleeds. Amyloid-related pathology and hypertensive cerebral small vessel disease have synergistic effects on the progression of lobar microbleeds [15]. There is also evidence from animal studies for an age-independent effect of tubulointerstitial kidney damage on brain $A \beta$ accumulation, which can be reinforced by the co-presence of cerebral microhemorrhages [16].

This case presented evidence of arteriosclerosis, but a statin rather than an antiplatelet agent was administered to prevent future ischemic cerebrovascular events due to the high risk for ICH in CAA, particularly in patients with severe microbleed burden or a history of lobar hemorrhage. A prospective cohort study found that aspirin induced an approximately fourfold increase in the risk for both ICH occurrence (when $\geq 5$ microbleeds are present) and recurrence in CAA [17]. In addition, we administered several antihypertensive agents to control blood pressure because active antihypertensive treatments have been shown to reduce the risk of CAA-related and hypertension-related ICH (by 77\% and 46\%) [18]. Currently, there are insufficient data to recommend against statin agents so they were administered as preventative therapy [19].

We analyzed PBMC counts to assess immune status and the results showed an increased CD19+ B-cell fraction among PBMCs at baseline and a gradual return to normal over the course of treatment. It is generally believed that the CD19+ B-cell fraction reflects the activity of humoral immunity, which is strongly involved in CAA-I pathogenesis. Several studies have shown that anti-amyloid autoantibodies are increased in the CSF during the acute phase of inflammation and return to control levels during remission $[20,21]$. Unfortunately, this case was not tested for CSF anti-amyloid antibodies. However, it should be 
noted that the diagnostic value of CSF anti-amyloid antibodies has not been definitively demonstrated. Alternatively, we conducted dynamic monitoring of PBMC CD19+ B-cell count to guide the tapering regime of oral prednisone, leading to substantial clinical and radiographic improvement. The patient was satisfied with such individualized corticosteroid treatments.

Management of CAA-I is challenging and should be individualized, especially for patients with preexisting risk factors for ischemic cerebrovascular diseases. Corticosteroid treatment is effective for reducing inflammation related to CAA. Dynamic monitoring of the CD19+ B-cell number among PBMCs may be useful for guiding corticosteroid treatment to achieve optimal therapeutic response with minimal side effects.

\section{Abbreviations}

APOE: Apolipoprotein E, ADL: Activities of Daily Living, AB: Amyloid- $\beta, C T$ : Computed tomography, CRP: Creactive protein, CSF: Cerebrospinal fluid, CAA: Cerebral amyloid angiopathy, CAA-l: Cerebral amyloid angiopathy-related inflammation, DWl; Diffusion-weighted imaging, ESR: Erythrocyte Sedimentation Rate, FLAIR: Fluid-attenuated inversion recovery, IVMP: Intravenous methylprednisolone, MMSE: Mini-Mental State Examination, MoCA: Montreal Cognitive Assessment, MRA: Magnetic Resonance Angiography, MRI: Magnetic Resonance Imaging (MRI), PBMC: Peripheral Blood Mononuclear Cell, SWI: Susceptibility Weighted Imaging, TGAb: thyroglobulin antibody, TPOAb: thyroid peroxidase antibody, T2WI: T2-weighted images, TIWI: T1-weighted images

\section{Declarations}

\section{Ethics approval and consent to participate}

Approval from the Ethical Committee of Affiliated Hospital of Guizhou Medical University to conduct this study has obtained.

\section{Consent for publication}

Written informed consent was obtained from the patient's legal guardian for the publication of this case report.

\section{Availability of data and material}

Not applicable.

\section{Competing interests}

The authors declare that they have no competing interests.

\section{Funding}


This study was supported by the Department of Science and Technology of Guiyang City, Guizhou Province, China [grant number (2017) 5-5].

\section{Authors' contributions}

HD: study concept and manuscript revision. LY: manuscript writing. L-CT: manuscript revision and editing. G-SP, Z-AN and W-XL: data collections.

\section{Acknowledgments}

The authors would like to thank Guofeng Wu and YuanHong Mao, Department of Emergency, Affiliated Hospital of Guizhou Medical University, Guiyang City, Guizhou Province, and HongYu Zhou, Qin Du, ZiYan Shi, Department of Neurology, HuiJiao Chen, Department of Pathology, West China Hospital, Sichuan University, Chengdu City, Sichuan Province, China, for their contributions in the technical assistance of brain biopsy and pathological examinations.

\section{References}

1. Kinnecom C, Lev MH, Wendell L, Smith EE, Rosand J, Frosch MP, et al. Course of cerebral amyloid angiopathy-related inflammation. Neurology. 2007; 68:1411-1416.

2. Chung KK, Anderson NE, Hutchinson D, Synek B, Barber PA. Cerebral amyloid angiopathy re-lated inflammation: three case reports and a review. J Neurol Neurosurg Psychiatry. 2011; 82:20-26.

3. Auriel E, Charidimou A, Gurol ME, Ni J, Van Etten ES, Martinez-Ramirez S, et al. Validation of clinicoradiological criteria for the diagnosis of cerebral amyloid angiopathy-related inflamma-tion. JAMA Neurol. 2016; 73:197-202.

4. Schrag M, McAuley G, Pomakian J, Jiffry A, Tung S, Mueller C, et al. Correlation of hy-pointensities in susceptibility-weighted images to tissue histology in dementia patients with cere-bral amyloid angiopathy: a postmortem MRI study. Acta Neuropathol. 2010; 119:291-302.

5. Warren JD, Schott JM, Fox NC, Thom M, Revesz T, Holton JL, et al. Brain biopsy in dementia. Brain. $2005 ; 128: 2016-2025$.

6. Cupino TL, Zabel MK. Alzheimer's silent partner: cerebral amyloid angiopathy. Transl Stroke Res. $2014 ; 5: 330-337$.

7. Vinters HV. Cerebral amyloid angiopathy. A critical review. Stroke. 1987; 18:311-324.

8. Verbeek MM, Kremer BP, Rikkert MO, Van Domburg PH, Skehan ME, Greenberg SM. Cere-brospinal fluid amyloid beta(40) is decreased in cerebral amyloid angiopathy. Ann Neurol. 2009; 66:245-249.

9. Kester MI, Goos JD, Teunissen CE, Benedictus MR, Bouwman FH, Wattjes MP, et al. Associa-tions between cerebral small-vessel disease and Alzheimer disease pathology as measured by cerebrospinal fluid biomarkers. JAMA Neurol. 2014; 71:855-862. 
10. Rannikmäe K, Samarasekera N, Martînez-Gonzâlez NA, Al-Shahi Salman R, Sudlow CL. Ge-netics of cerebral amyloid angiopathy: systematic review and meta-analysis. J Neurol Neurosurg Psychiatry. 2013; 84:901-908.

11. Biffi A, Sonni A, Anderson CD, Kissela B, Jagiella JM, Schmidt H, et al. Variants at APOE influence risk of deep and lobar intracerebral hemorrhage. Ann Neurol. 2010; 68:934-43.

12. Allen N, Robinson AC, Snowden J, Davidson YS, Mann DM. Patterns of cerebral amyloid an-giopathy define histopathological phenotypes in Alzheimer's disease. Neuropathol Appl Neurobiol. 2014; 40:136-148.

13. Chung YC, Kruyer A, Yao Y, Feierman E, Richards A, Strickland S, et al. Hyperhomocyste-inemia exacerbates Alzheimer's disease pathology by way of the $\beta$-amyloid fibrinogen interaction. J Thromb Haemost. 2016; 14:1442-1452.

14. Chong JY, Rowland LP, Utiger RD. Hashimoto encephalopathy: syndrome or myth? Arch Neurol. 2003; 60:164-171.

15. Kim YJ, Kim HJ, Park JH, Kim S, Woo SY, Kwak KC, et al. Synergistic effects of longitudinal amyloid and vascular changes on lobar microbleeds. Neurology. 2016; 87:1575-1582.

16. Pirici D, Stanaszek L, Garz C, Niklass S, Heinze HJ, Kalinski T, et al. Common Impact of Chronic Kidney Disease and Brain Microhemorrhages on Cerebral A $\beta$ Pathology in SHRSP. Brain Pathol. 2017; 27:169-180.

17. Biffi A, Halpin A, Towfighi A, Gilson A, Busl K, Rost N, et al. Aspirin and recurrent intracere-bral hemorrhage in cerebral amyloid angiopathy. Neurology. 2010; 75:693-698.

18. Arima H, Tzourio C, Anderson C, Woodward M, Bousser MG, MacMahon S, et al. Effects of perindopril-based lowering of blood pressure on intracerebral hemorrhage related to amyloid angiopathy: the PROGRESS trial. Stroke. 2010; 41:394-396.

19. Hemphill JC 3rd, Greenberg SM, Anderson CS, Becker K, Bendok BR, Cushman M, et al. Guidelines for the Management of Spontaneous Intracerebral Hemorrhage: A Guideline for Healthcare Professionals From the American Heart Association/American Stroke Association. Stroke. 2015; 46:2032-2060.

20. Piazza F, Greenberg SM, Savoiardo M, Gardinetti M, Chiapparini L, Raicher I, et al. Anti-amyloid $\beta$ autoantibodies in cerebral amyloid angiopathy-related inflammation: implications for amyloidmodifying therapies. Ann Neurol. 2013; 73:449-458.

21. Carmona-Iragui M, Fernández-Arcos A, Alcolea D, Piazza F, Morenas-Rodriguez E, Antón-Aguirre S, et al. Cerebrospinal Fluid Anti-Amyloid- $\beta$ Autoantibodies and Amyloid PET in Cere-bral Amyloid Angiopathy-Related Inflammation. J Alzheimers Dis. 2016; 50:1-7.

\section{Figures}



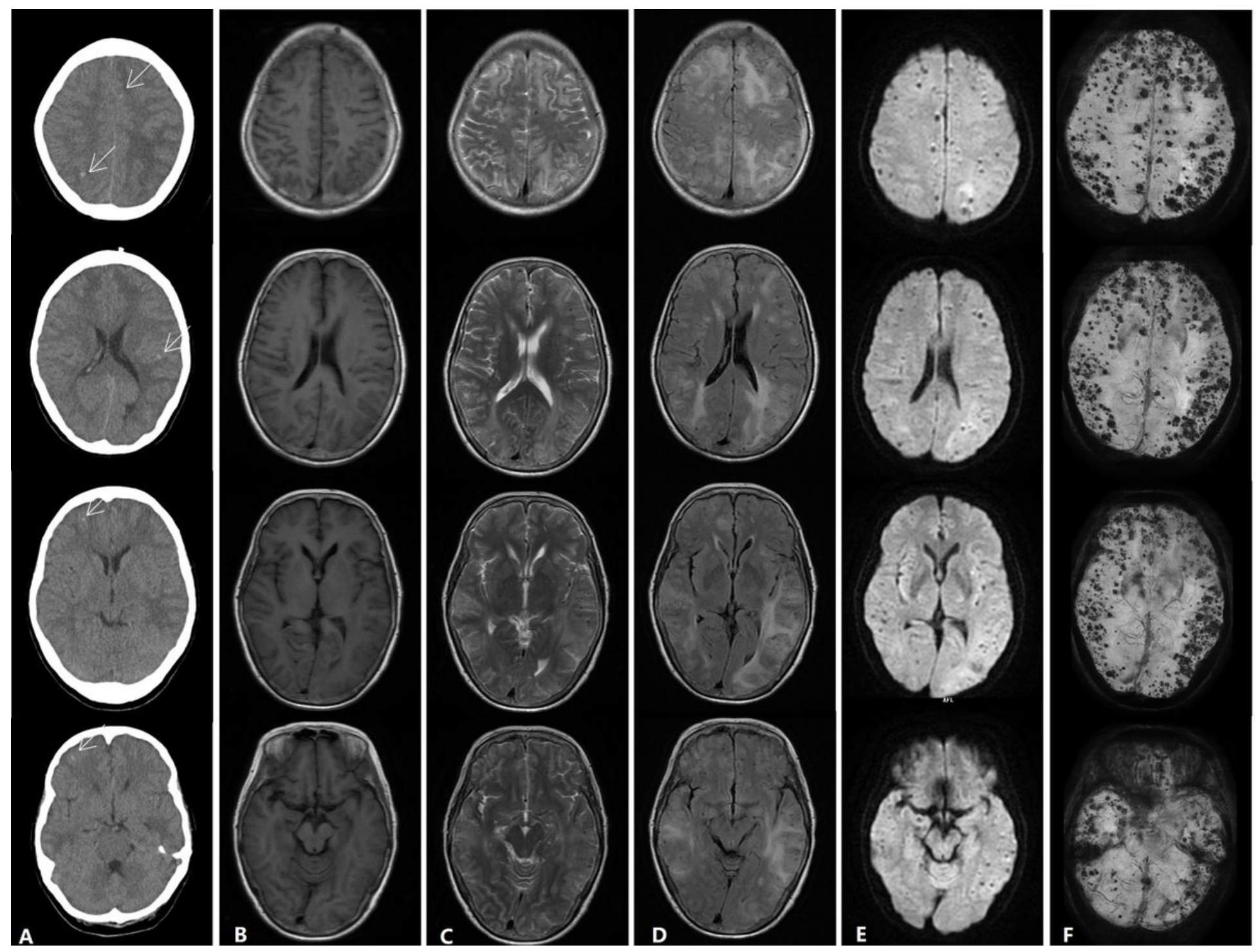

Figure 1

Findings of axial brain CT plain scans and brain MRI scans at the time of admission. Brain CT images showed multiple punctiform hyperdensities (arrows) in the bilateral lobes (A). Brain MRI scans showed extensive hypointense lesions on T1WI (B) and hyperintense lesions on both T2WI (C) and FLAIR (D) images in the bilateral cerebral hemispheres. SWI revealed multiple lobar microbleeds in the bilateral lobes and cerebellum (E). CT: Computed tomography, MRI: magnetic resonance imaging, SWI: susceptibility-weighted imaging, T1WI: T1-weighted imaging, T2WI: T2-weighted imaging. 


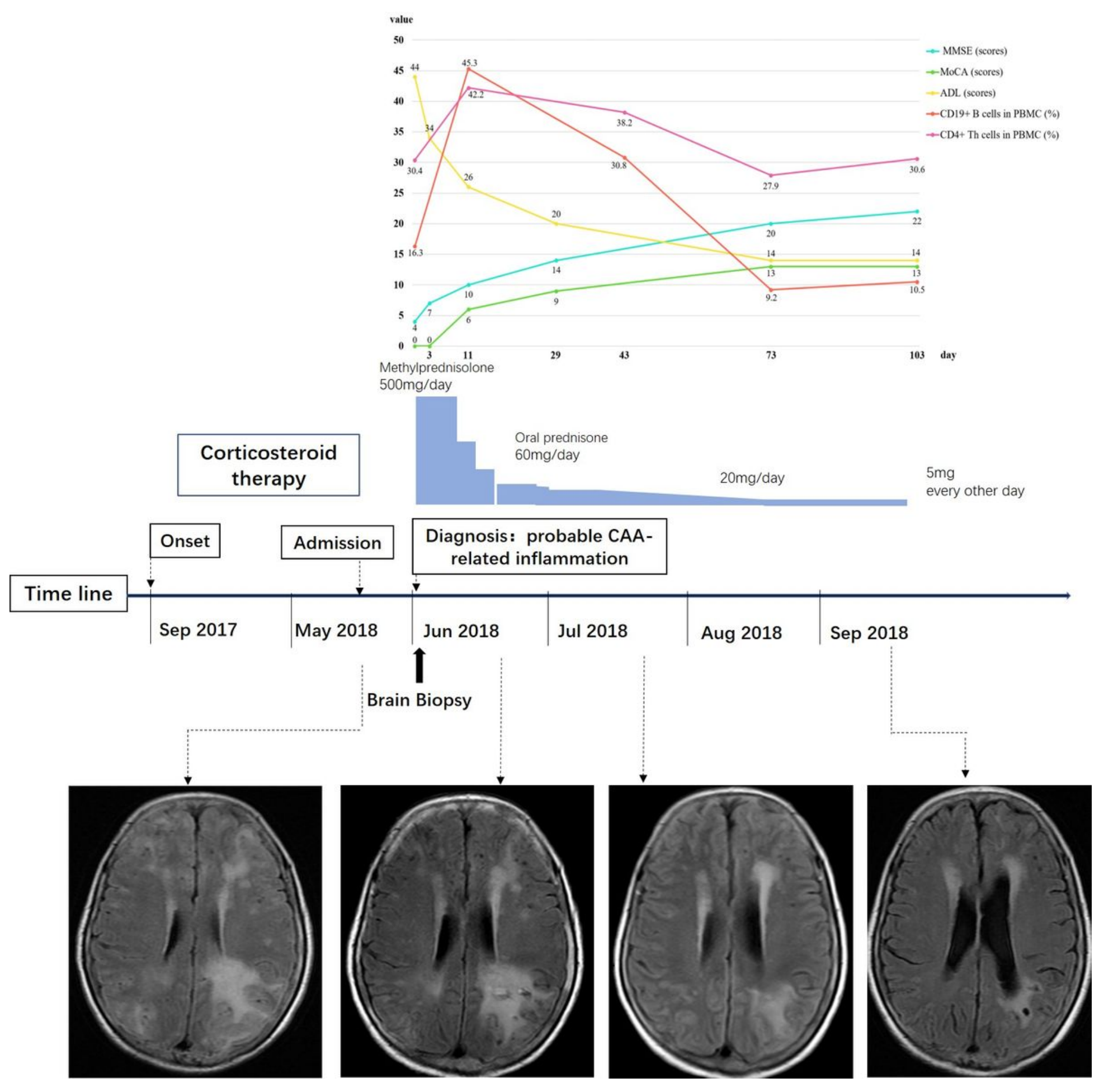

Figure 2

Longitudinal changes in CD19+B-cell and CD4+Th-cell fractions among PBMCs, lesions on brain MRI, cognitive function, and daily living capacity during corticosteroid treatment. ADL: Activities of Daily Living, MMSE: Mini-Mental State Examination, MoCA: Montreal Cognitive Assessment, PBMC: Peripheral blood mononuclear cells.

\section{Supplementary Files}


This is a list of supplementary files associated with this preprint. Click to download.

- renamed71880.pdf 